Original Paper

\title{
Enzyme Inhibitory Activity of Ovomucoid Extracted Using a Carboxypeptidase Y-Immobilized Membrane
}

\author{
Youji SHIMAZAKI ${ }^{* 1,2}$, Kosuke TANAKA $^{2}$, Keisuke SAKATA $^{2}$ \\ ${ }^{I}$ Graduate School of Science and Engineering (Science), Ehime University, 2-5 Bunkyo-cho, Matsuyama 790-8577, Japan \\ ${ }^{2}$ Department of Chemistry, Faculty of Science, Ehime University, 2-5 Bunkyo-cho, Matsuyama 790-8577, Japan
}

\begin{abstract}
Reversible inhibition of enzymes is caused by association and dissociation between enzymes and inhibitors. Therefore, reversible inhibitors can be trapped and extracted using enzyme-inhibitor interaction. The purpose of this study was to establish a method in which the reversible inhibitors retaining the original inhibitory activities are extracted from a single drop of biological sample using the enzyme-inhibitor interaction on the surface of a membrane. A membrane-immobilized carboxypeptidase Y (CPY) was produced after the biotinylated CPY was bound to the avidin separated by nondenaturing electrophoresis, transferred to a polyvinylidene fluoride and stained by Ponceau S. Ovomucoid, possessing reversible CPY inhibitory activity, was trapped and extracted from a single drop of egg white and isolated using the membrane-immobilized CPY. The isolated ovomucoid using this membrane-immobilized CPY possessed a feature that more than $85 \%$ of the relative carboxylesterase activity was suppressed. The results indicate that ovomucoid retaining enzyme inhibitory activities can be isolated from a single drop of egg white sample using enzyme-immobilized membrane.
\end{abstract}

Keywords: Carboxypeptidase Y; Carboxylesterase; Ovomucoid; Inhibitory activity; Reversible inhibitor

\section{Introduction}

Interactions between enzymes and inhibitors depend on regulation of the enzyme activities. Reversible inhibition of enzyme activity is primarily caused by interactions such as association and dissociation between enzymes and inhibitors [1-3]. Ovomucoid, from egg white, is a serine protease inhibitor [4-6] that reversibly inhibits the activity of the serine protease trypsin [7]. The reversible inhibition by ovomucoid can be examined in the presence of the immobilized serine protease, because activity changes of serine protease, such as trypsin and carboxypeptidase $\mathrm{Y}$ (CPY), were analyzed by the combined methods of matrix-assisted laser desorption/ionization time-of-flight mass spectrometry (MALDI-TOF MS) and the analysis of the enzyme immobilized on supports [8,9]. Based on the association and dissociation between ovomucoid and trypsin, ovomucoid is purified using trypsin-sepharose affinity chromatography [10]. Thus, ovomucoid can be trapped and extracted based on the interaction properties

${ }^{*}$ Corresponding author: Youji SHIMAZAKI

Tel: +81-89-927-9617; Fax: +81-89-927-9590

E-mail: shimazaki.yoji.my@ehime-u.ac.jp between ovomucoid and CPY.

Noncovalent intermolecular interaction on a membrane can be applied to the isolation molecules from a single drop of biological samples. Because interactions between antigen and antibody occur on the surface of a membrane, antigens within a single drop of biological sample can be trapped and extracted by membrane-immobilized antibodies $[11,12]$. Also, because other interactions, such as that of enzyme-inhibitor, can be performed on the membrane, ovomucoid can be trapped within a single drop of egg white and can be extracted using membrane-immobilized CPY.

In addition, the extracted ovomucoid can retain the intact properties, such as its interaction with other enzymes. Because the dominant force for binding serine protease and ovomucoid has been reported to be hydrophobic interactions [13], the extracted ovomucoid can interact with the hydrophobic catalytic sites of other enzymes. Because carboxylesterase possesses a hydrophobic catalytic pocket site [14], its activity can be inhibited by the extracted

Received: 3 November 2017

Accepted: 17 December 2017

J-STAGE Advance Published: 29 December 2017

DOI: $10.15583 /$ jpchrom.2017.020 
ovomucoid. However, the amount of ovomucoid extracted from membrane-immobilized CPY is considered to be a very small quantity. Thus, a highly sensitive detection method is required for analyzing enzyme activity. We have previously reported that the activity of 0.0014-0.14 Unit $/ \mathrm{mL}$ of carboxylesterase can be examined using a combinational method of a fluorescent substrate and spectrofluorometry [11]. This method examines whether the ovomucoid trapped and extracted by membraneimmobilized CPY possesses inhibitory activity for carboxylesterase.

The purpose of this study is to establish a method to extract reversible inhibitors from a single drop of egg white using the interaction between enzyme and inhibitor on the surface of membrane-immobilized enzyme. This method can be used to isolate reversible enzyme inhibitors while still fully retaining the original inhibitory activities of the said inhibitors.

\section{Experimental}

\subsection{Chemicals and sample preparation}

Carboxypeptidase Y (CPY) from baker's yeast (S. cerevisiae), adrenocorticotropic hormone (ACTH) from porcine pituitary, avidin from egg white and 4-methylumbelliferyl acetate (4-MA) were purchased from Sigma Aldrich (St. Louis, MO, USA). Polyvinylidene fluoride (PVDF) membrane, biotin Labeling Kit- $\mathrm{NH}_{2}$ and acrylamide were purchased from Merck-Millipore (Bedford, MA, USA), Dojindo Laboratories (Kumamoto, Japan) and Daiichi Pure Chemical (Osaka, Japan), respectively. Rabbit anti-chicken ovomucoid polyclonal antibody and goat anti-rabbit IgG-HRP were purchased from Flarebio biotech LLC. (College Park, MD, USA) and Santa Cruz Biotechnology INC. (Dallas, TX, USA), respectively. All other reagents were purchased from Wako Pure Chemicals (Osaka, Japan) or Nacalai Tesque (Osaka, Japan). A freshly-laid egg was used for the sample preparation. After the egg white was separated from the yolk, it was two-fold diluted with $0.1 \mathrm{M}$ Tris- $\mathrm{HCl}$ solution ( $\mathrm{pH}$ 7.0). The soluble fraction of the egg white was obtained by centrifuging $(10,000 \times \mathrm{g})$ for $30 \mathrm{~min}$.

\subsection{Analysis of membrane-immobilized CPY activity by MALDI-TOF MS}

Biotinylated CPY on membrane-immobilized avidin was produced following previously published methods [15]. Briefly, sucrose was added to avidin (Sigma-Aldrich, St. Louis, MO, USA) to a concentration of $40 \%$. Then, $5 \mu \mathrm{L}$ of $10 \mu \mathrm{g} / \mu \mathrm{L}$ avidin was subjected to nondenaturing electrophoresis. After separation, protein was transferred to a polyvinylidene fluoride (PVDF) membrane [15]. After the avidin was separated, blotted onto the membrane, and stained, the 4-mm square spot was cut off. After the biotinylated CPY was produced using the Biotin Labeling Kit- $\mathrm{NH}_{2}, 12 \mu \mathrm{L}$ of $1.6 \mu \mathrm{M}$ CPY was added to the membrane-immobilized avidin and incubated for $1 \mathrm{~h}$ to produce membrane-immobilized CPY. For enzymatic digestion of porcine ACTH, $10 \mu \mathrm{L}$ of $0.2 \mathrm{mM} \mathrm{ACTH}$ solution ( $\mathrm{pH}$ 7.0) was added to the membrane-immobilized CPY and incubated for 0-60 min.

In order to examine inhibition of protease activity within the soluble fraction of the egg white or the purified ovomucoid, $10 \mu \mathrm{L}$ of the soluble fraction in the egg white or $10 \mu \mathrm{L}$ of $0.36 \mathrm{mM}$ purchased ovomucoid was added to the membrane and incubated for $30 \mathrm{~min}$. Additionally, 10 $\mu \mathrm{L}$ of $0.2 \mathrm{mM} \mathrm{ACTH}$ solution containing in the soluble fraction or the purified ovomucoid was added to the membrane, and incubated for $30 \mathrm{~min}$. After washing the membrane with $0.1 \mathrm{M}$ Tris- $\mathrm{HCl}(\mathrm{pH} 7.0), 10 \mu \mathrm{L}$ of $0.2 \mathrm{mM}$ ACTH solution in the absence of the soluble fraction or ovomucoid was added to the membrane and incubated for $30 \mathrm{~min}$ in order to examine enzymatic digestion. After incubation, the solution containing ACTH on the membrane was collected and mixed with $1 \mu \mathrm{L}$ of $25 \%$ TFA. Then, 1 $\mu \mathrm{L}$ of sample and a matrix solution containing $20 \mathrm{mg} / \mathrm{mL}$ $\alpha$-cyano-4-hydroxycinnamic acid (CHCA) and $0.1 \%$ trifluoroacetic acid (TFA) in $60 \%$ acetonitrile was analyzed via MALDI-TOF MS according to previously published methods [16,17]. MALDI-TOF MS analysis was performed using a Voyager-DE PRO (Applied Biosystems, Framingham, MA, USA).

\subsection{Extraction of the proteins bound to the} membrane-immobilized CPY and purification of ovomucoid

Fig. 1 shows schematic fractionation protocol of egg white proteins. Ten $\mu \mathrm{L}$ of the soluble fraction of egg white was added to the membrane-immobilized CPY or avidin (control) and incubated for $2 \mathrm{~h}$. After washing the membrane with $0.1 \mathrm{M}$ Tris- $\mathrm{HCl}$ ( $\mathrm{pH} 7.0), 10 \mu \mathrm{L}$ of $0.20 \mathrm{M}$ $\mathrm{KCl}$ solution containing $0.1 \mathrm{M}$ Tris- $\mathrm{HCl}(\mathrm{pH} 7.0)$ was added to the membrane and incubated for $30 \mathrm{~min}$. The $\mathrm{KCl}$ solution was thus collected. After the collected solution was diluted with $20 \mu \mathrm{L}$ of $\mathrm{H}_{2} \mathrm{O}$, the concentration of $\mathrm{KCl}$ in the collected solution was reduced by ultrafiltration (Microcon, 3,000 cut-off filter, Millipore, Darmstadt, Germany) to obtain the CPY fraction. For further purification of ovomucoid from the CPY fraction according to a previously published method $[18,19]$, the proteins in the CPY fraction were precipitated by slowly adding $100 \%$ ethanol to the final concentration of $43 \%$, and centrifuged at $10,000 \times \mathrm{g}$ for $20 \mathrm{~min}$ at $4{ }^{\circ} \mathrm{C}$ to obtain the supernatant (S1) and the precipitate (P1). The ethanol concentration of the extract was increased to $61 \%$ (final) by slowly adding $100 \%$ ethanol to $\mathrm{S} 1$. The solution was kept at $4^{\circ} \mathrm{C}$ overnight and then centrifuged at $10,000 \times \mathrm{g}$ for $20 \mathrm{~min}$ at the same temperature to obtain the supernatant (S2) and the 
precipitate (P2). After removing ethanol in S2 by ultrafiltration, $\mathrm{S} 2$ was adjusted to $\mathrm{pH} 4.0$ using citric acid, heat-treated at $65^{\circ} \mathrm{C}$ for $20 \mathrm{~min}$, and centrifuged at 10,000 $\times \mathrm{g}$ for $30 \mathrm{~min}$ at $4{ }^{\circ} \mathrm{C}$ to obtain the supernatant (S3) and the precipitate ( $\mathrm{P} 3$ ). After neutralizing $\mathrm{S} 3$, the proteins in $\mathrm{S} 3$ were precipitated by adding $60 \%$ polyethylene glycol to the final concentration of $30 \%$ to obtain S4 fraction. The precipitate fractions ( $\mathrm{P} 1, \mathrm{P} 2, \mathrm{P} 3$ and $\mathrm{S} 4)$ were dissolved in $10 \mu \mathrm{L}$ of $0.1 \mathrm{M}$ Tris- $\mathrm{HCl}(\mathrm{pH} 7.0)$.

\section{Egg soluble proteins}

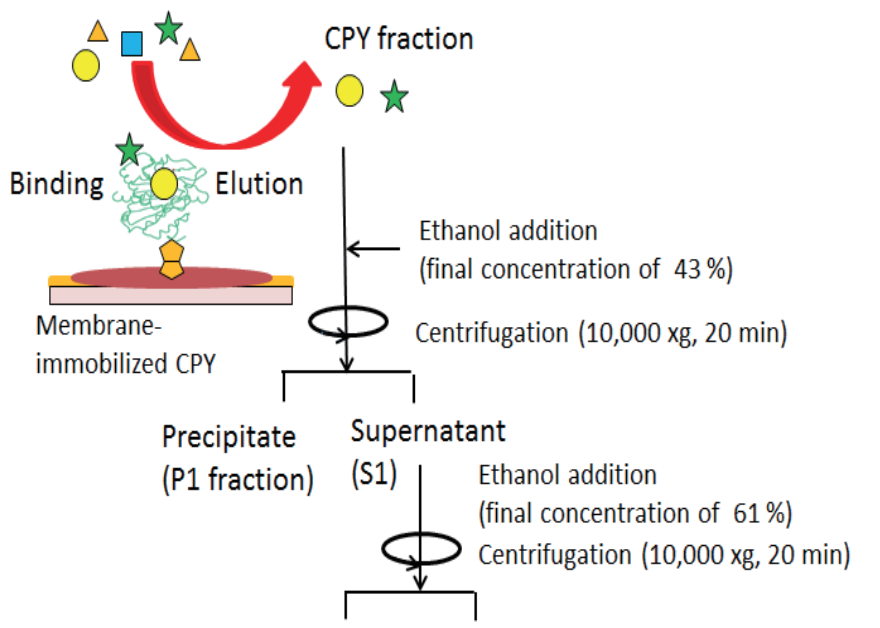

Precipitate Supernatant

(P2 fraction) (S2) Utrafiltration

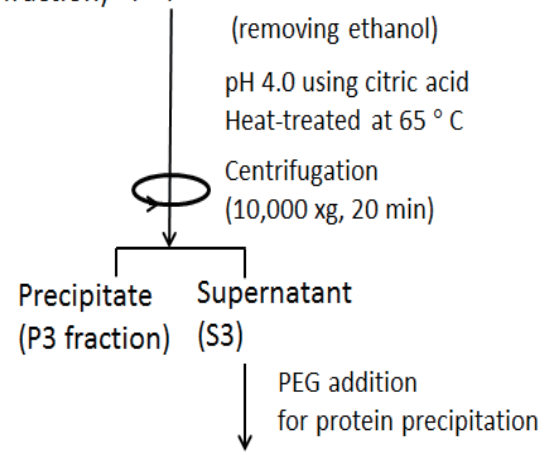

S4 fraction

Fig. 1. Schematic fractionation protocol of egg white proteins.

Then, inhibition of esterase activity in each fraction (P1, P2, P3 and S4) was examined. For interaction analysis of esterase activity, after $10 \mu \mathrm{L}$ of $0.36 \mathrm{mM}$ purchased ovomucoid was added to the membrane-immobilized CPY, the ovomucoid bound to the membrane-immobilized CPY was extracted and treated as mentioned above. Then, inhibition of esterase activity was examined. Furthermore, the extracted and purified proteins in the factions (P1, P2, P3 and S4) or the purchased ovomucoid were separated by SDS-PAGE ( $9 \%$ acrylamide).

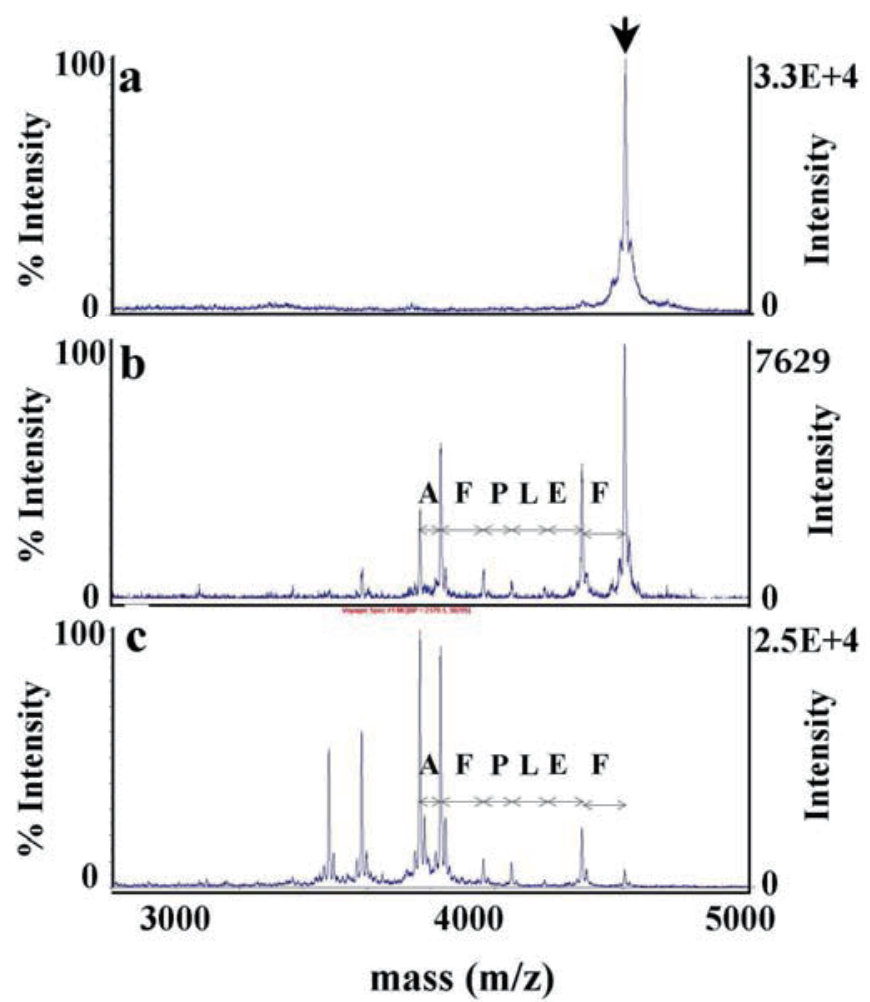

Fig. 2. MALDI-TOF MS spectra of porcine ACTH before (a) and after ( $b$ and $c$ ) digestion using membrane-immobilized CPY. The peaks of $\mathrm{m} / \mathrm{z} 4568.15,4421.16,4292.72,4177.80$, 4082.60, 3935.03 and 3863.84 were obtained after the digestion using CPY for $30 \mathrm{~min}$ (b) and $60 \mathrm{~min}$ (c). The mass differences may correspond to the amino acid sequence AFPLEF, the carboxyl-terminal end of porcine ACTH. Arrow indicates original ACTH before digestion by membrane-immobilized CPY.

\subsection{Measurement of esterase activity using a spectrofluorometer}

For the semi-quantitative analysis of esterase activity, 4-MA (1 $\mathrm{mg})$ was dissolved in $0.5 \mathrm{~mL}$ $N, N$-dimethylformamide. The solution was diluted to the final concentration of $0.46 \mu \mathrm{M}$ with $0.1 \mathrm{M}$ phosphate buffer (pH 6.9) (4-MA solution). After $110 \mu \mathrm{L}$ of the 4-methylumbelliferyl acetate solution was added to the samples containing $0.6 \mathrm{nM}$ carboxylesterase in the absence or presence of the fractions (CPY, P1, P2, P3 or S4) obtained from egg white, the changes in fluorescence within 3 min were measured at an excitation wavelength of $360 \mathrm{~nm}$ and an emission wavelength of $450 \mathrm{~nm}$ using a spectrofluorometer, FP-8200 from JASCO (Tokyo, Japan). Enzyme kinetics was calculated by the changes in fluorescence intensity per unit time. For quantitative inhibition analysis, the changes in fluorescence by 0.1-6 $\mathrm{nM}$ carboxylesterase in the presence of $0.36 \mu \mathrm{M}$ purchased ovomucoid were compared with those in the absence of the ovomucoid. The data were analyzed using a Student's $t$-test. 

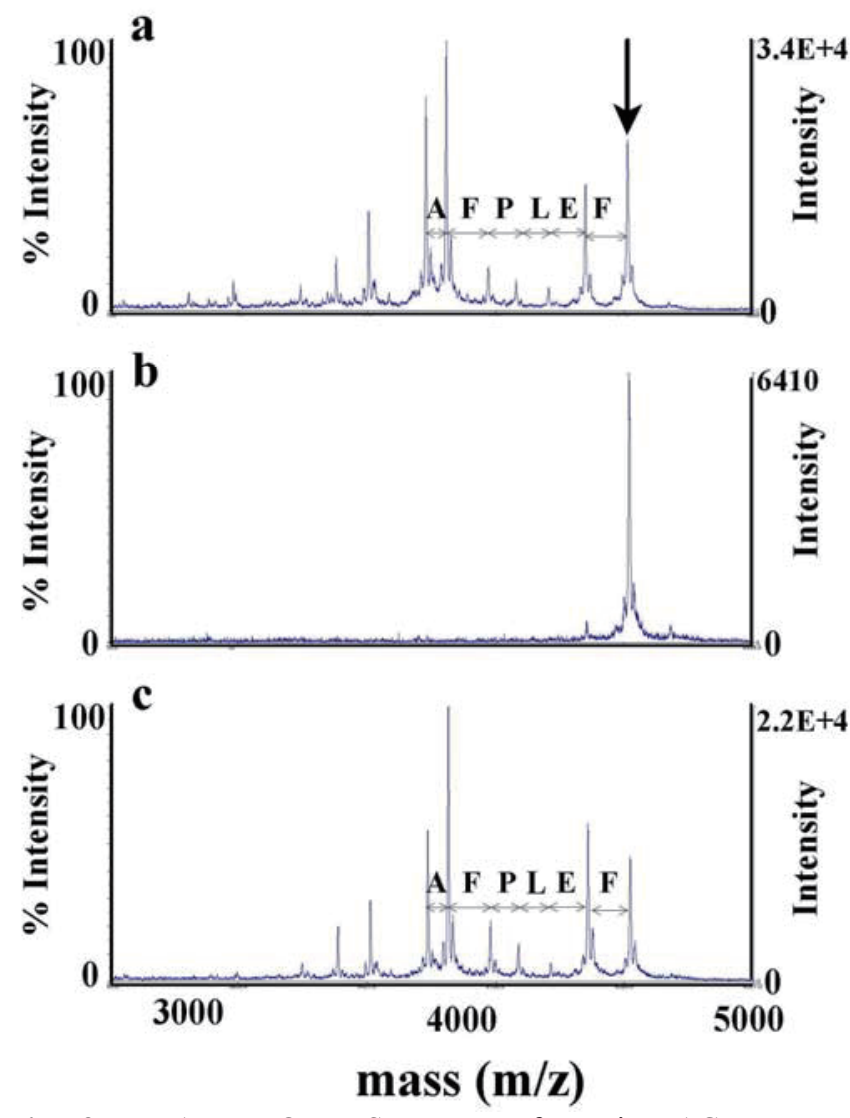

Fig. 3. MALDI-TOF MS spectra of porcine ACTH was incubated on membrane-immobilized CPY in the absence (a) and presence (b) of $10 \mu \mathrm{L}$ the soluble fraction of egg white, and then the inhibitors were washed away from the membrane (c). The mass differences may correspond to the amino acid sequence AFPLEF. Arrow indicates the peak of original ACTH.

\section{Results and discussion}

3.1. Assay of membrane-immobilized CPY activity using MALDI-TOF MS

Fig. 2 shows the MALDI-TOF MS spectra of porcine ACTH before (a) and after (b and c) it was digested using the membrane-immobilized CPY. The peaks of $\mathrm{m} / \mathrm{z}$ 4568.15, 4421.16, 4292.72, 4177.80, 4082.60, 3935.03 and 3863.84 were obtained after the digestion by CPY (Fig. 2b and $2 \mathrm{c}$ ). The mass differences may correspond to the amino acid sequence, AFPLEF, the carboxyl-terminal end of porcine $\mathrm{ACTH}$, indicative of the hormone being digested by the membrane-immobilized CPY. And, ACTH digestion by the membrane-immobilized CPY for $30 \mathrm{~min}$, the peaks at $\mathrm{m} / \mathrm{z} 4568.15$ and 4421.16 were clearly obtained (Fig. 2b), whereas they were decreased and other peaks at $\mathrm{m} / \mathrm{z}$ 3935.03 and 3863.84 were clearly obtained after the digestion for $60 \mathrm{~min}$ (Fig. 2c).

\subsection{Reversible inhibition of CPY activity on the membrane} by egg white protein
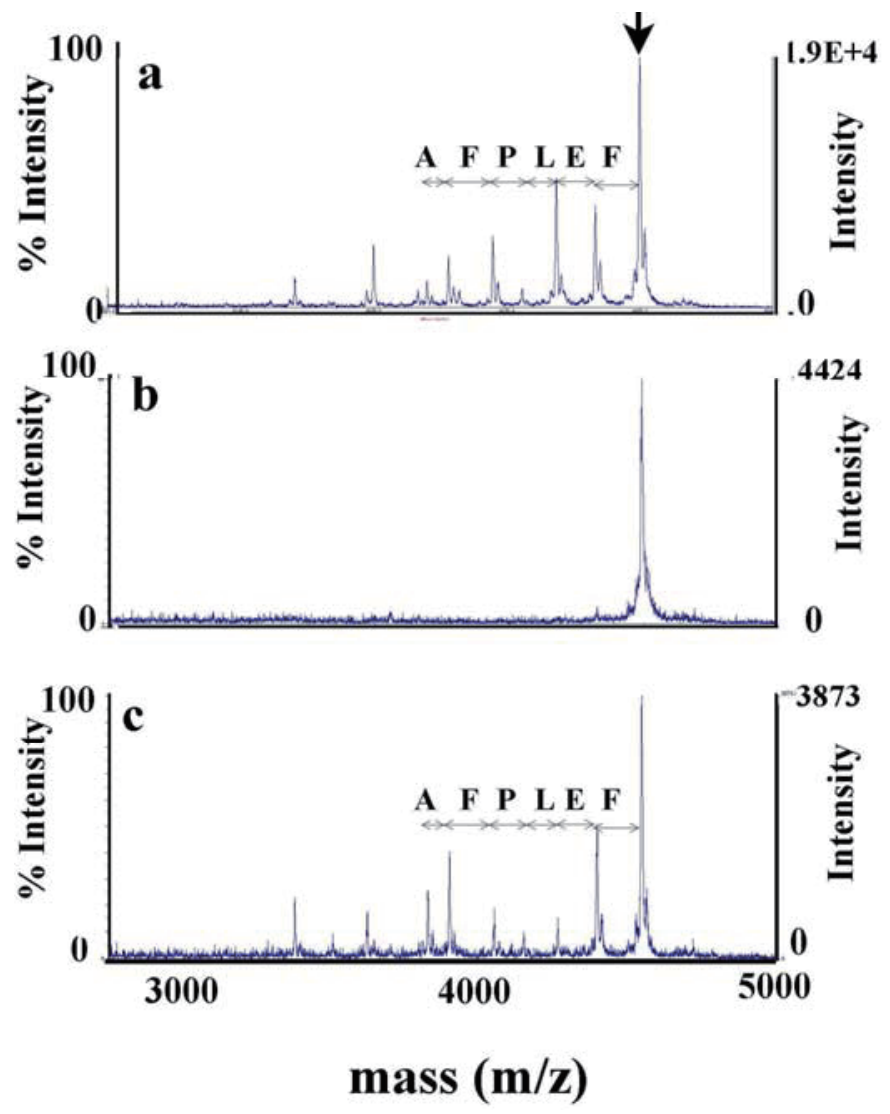

Fig. 4. MALDI-TOF MS spectra of porcine ACTH was also incubated on membrane-immobilized CPY in the absence (a) and presence (b) of ovomucoid $(10 \mu \mathrm{L}$ of $0.36 \mathrm{mM})$, and ovomucoid was washed away from the membrane (c). The mass differences may correspond to the amino acid sequence AFPLEF. Arrow indicates the peak of original ACTH.

The digested peaks were obtained when porcine ACTH was incubated in the absence of the soluble fraction of egg white (Fig. 3a), whereas they were not obtained when it was incubated in the presence of $10 \mu \mathrm{L}$ of the soluble fraction of egg white (Fig. 3b), but the peaks were obtained after the soluble fraction of egg white was washed away from the membrane (Fig. 3c). The mass differences as seen in Fig. 3c may also correspond to the amino acid sequence AFPLEF; thereby indicating that CPY enzymatic activity was recovered. The results indicate that the soluble fraction of egg white contains reversible inhibitors of CPY activity, which can be caused by association and dissociation between inhibitors and CPY on the membrane. It has been reported that a protease inhibitor of duck egg protein, ovomucoid is purified by trypsin-sepharose [12]. Thus, the same protein of soluble fraction of chicken egg white can be bound to the membrane-immobilized CPY, and can be extracted, because of association and dissociation between ovomucoid and CPY on the membrane. Porcine ACTH was digested by the CPY membrane in the absence of the 
purchased ovomucoid (Fig. 4a), whereas it was not digested by the CPY membrane in the presence of the purchased ovomucoid (Fig. 4b). However, the CPY membrane regained the ability to digest $\mathrm{ACTH}$ after the ovomucoid was washed away from the membrane (Fig. 4c). These results indicated that ovomucoid acted as a reversible inhibitor of CPY activity, which can be caused by association and dissociation between ovomucoid and CPY on the membrane.

\subsection{Inhibition of carboxylesterase activity by soluble} fractions from egg white
Fig. 5a shows the relative esterase activity in the presence of the soluble proteins in egg white after trapping and extraction by membrane-immobilized CPY (CPY) or membrane-immobilized avidin (control). The carboxylesterase (ES) activity was significantly suppressed by the soluble proteins trapped and extracted by membrane-immobilized CPY, compared to those by control membrane. The soluble proteins extracted from egg white by the CPY or control membrane were separated by SDS-PAGE (Fig. 5b). Several protein bands were obtained after soluble proteins in egg white were trapped and extracted using the CPY or control membrane: the levels of $\mathbf{a}$
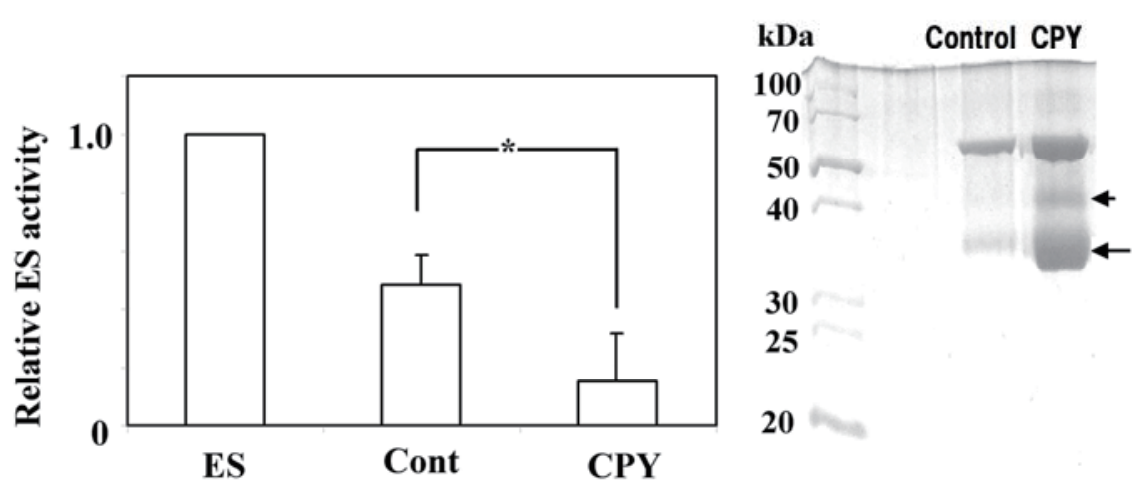

Fig. 5. (a) Relative esterase activity in the absence (ES) and presence of soluble proteins in egg white after trapping and extraction by membrane-immobilized CPY (CPY) or membrane-immobilized avidin (Cont; control). (b) SDS-PAGE analysis of the soluble proteins extracted from egg white by CPY or control. membrane. Specific protein bands at 38 $\mathrm{kDa}$ and $42 \mathrm{kDa}$ (arrows) were obtained after soluble proteins in egg white were trapped and extracted using the membrane- immobilized CPY. a

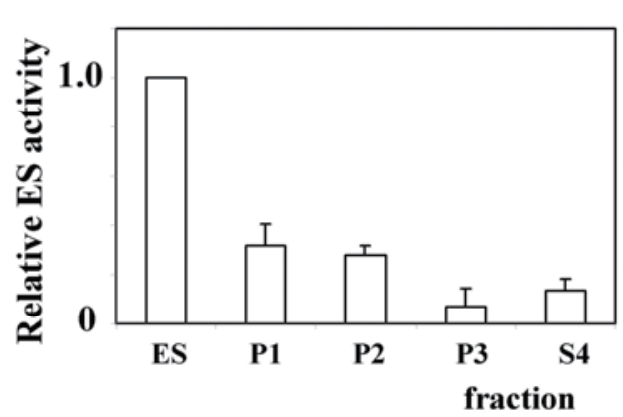

b

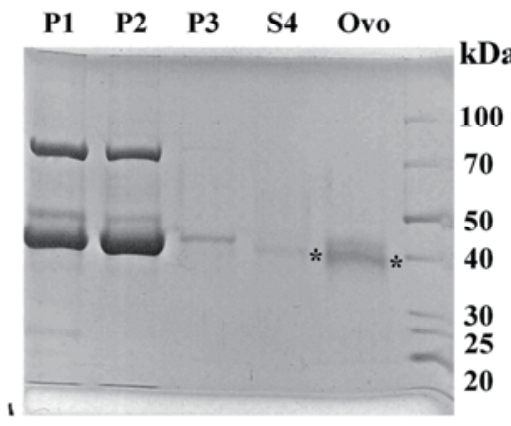

Fig. 6. (a) Relative esterase activity in the absence (ES) and presence of the fractionated proteins $(\mathrm{P} 1, \mathrm{P} 2, \mathrm{P} 3$ and S4 fractions) in egg white after extraction using membrane-immobilized CPY and fractionation. (b) SDS-PAGE analysis of proteins after soluble proteins were extracted using CPY and were fractionated. The purchased ovomucoid and protein band are indicated as Ovo and $*$, respectively.

a

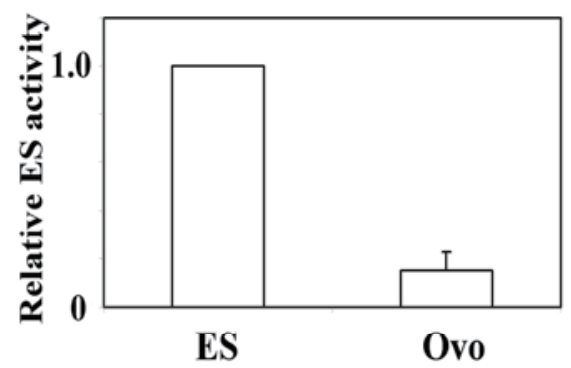

b

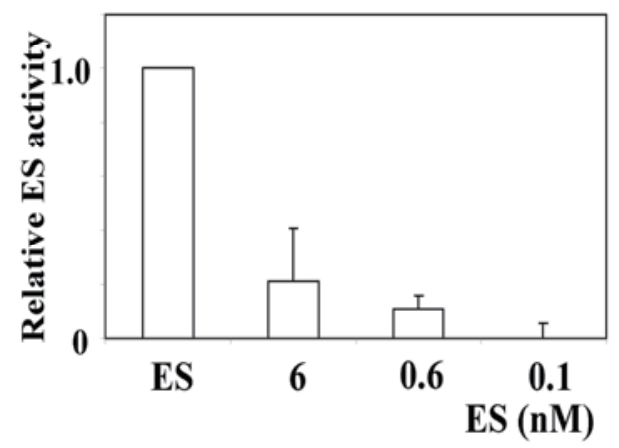

Fig. 7. (a) Relative esterase activity in the absence (ES) or presence of the purchased ovomucoid after trapping and extraction by membrane-immobilized CPY (Ovo). (b) Relative hydrolytic activity of $2.3 \mu \mathrm{M} \quad$ of 4-methylumbelliferyl acetate by 0.1-6 $\mathrm{nM}$ carboxylesterase in the presence of $0.36 \mu \mathrm{M}$ of the purchased ovomucoid. Relative esterase activity in the absence of the ovomucoid is indicated as ES. 
proteins at $38 \mathrm{kDa}$ and $42 \mathrm{kDa}$ clearly increased after the soluble proteins were trapped and extracted using the CPY membrane. The protein at $38 \mathrm{kDa}$ was immunoreacted to anti-chicken ovomucoid polyclonal antibody (data not shown). On the other hand, ES activity was also suppressed by the soluble proteins trapped and extracted by control membrane (Fig. 5a). However, small quantity of protein at $38 \mathrm{kDa}$ was obtained (Fig. 5b). Fig. 6a shows relative esterase activity in the absence (ES) and presence of the fractionated proteins (P1, P2, P3 and S4) in egg white after extraction using the CPY membrane and fractionation. The esterase activity was suppressed even after soluble proteins were fractionated from egg white (Fig. 6a). Fig. 6b shows SDS-PAGE of the proteins after soluble proteins were extracted using the CPY membrane (CPY) and fractionated. The P1, P2, P3 and S4 fractions contained the band at 38 $\mathrm{kDa}$, and, the molecular mass of the purchased ovomucoid was also $38 \mathrm{kDa}$ (Ovo in Fig. 6b). Thus, the band at $38 \mathrm{kDa}$ in the S4 fraction was thought to be ovomucoid after extraction using the CPY membrane and fractionation. The protein possessed inhibition of esterase activity as shown in Fig. 6a.

\subsection{Inhibition of carboxylesterase activity by ovomucoid}

Fig. 7a shows the relative esterase activity in the absence (ES) or presence of ovomucoid after trapping and extraction by membrane-immobilized CPY (Ovo). As the relative ES activity in the absence of the purified ovomucoid was referred to 1.0 , the suppression of the esterase activity after extraction by the CPY membrane was 0.15 (Fig. 7a). Fig. 7b shows the relative hydrolytic activity of $2.3 \mu \mathrm{M}$ of 4-methylumbelliferyl acetate by 0.1-6 nM esterase in the presence of $0.36 \mu \mathrm{M}$ purchased ovomucoid. The results indicate that $0.36 \mu \mathrm{M}$ of the ovomucoid can inhibit the activity of less than $0.6 \mathrm{nM}$ esterase. Ovomucoid has been reported to be purified using sepharose bound to serine protease such as trypsin [12]. The present study indicates that the activity of one of the serine protease, CPY bound to the membrane was reversibly inhibited by ovomucoid, and the protein was trapped and extracted by the CPY on the membrane. Furthermore, the extracted ovomucoid possessed carboxylesterase inhibitory activity. It has been reported that esterase activity in the homogenates of pancreas is inhibited by some inhibitors including ovomucoid [20]. On the other hand, the purified after soluble proteins in egg white were carboxylesterase was inhibited by the isolated ovomucoid in the present study. Because carboxylesterase contains hydrophobic amino acids at the catalytic pocket [14], the purified ovomucoid can interact with the hydrophobic sites of the catalytic pocket. Thus, ovomucoid possessing CPY inhibitory activity because of hydrolytic interaction also inhibited carboxylesterase activity. Ovomucoid has been purified from egg white using ion exchange chromatographic techniques [21,22]. For isolation and purification of ovomucoid from egg white, 1 liter of egg white homogenate is required. In this method, 10 microliter of egg white sample was used for isolation of ovomucoid. Furthermore, because the interaction between ovomucoid and CPY occur on the surface of the membrane, ovomucoid can be easily extracted after rinsing the membrane without impairing its function. Thus, the proposed method can be used to examine the native functions of isolated proteins using a single drop of biological sample.

\section{Conclusion}

Reversible inhibitors can associate and dissociate to the targeted enzymes. The present study indicates that a reversible inhibitor, ovomucoid, can be trapped and extracted from a single drop of egg white, using membrane-immobilized CPY. The extracted ovomucoid also possessed carboxylesterase inhibitory activity. The results indicate that intact-reversible-enzyme inhibitors from a single drop of biological samples can be isolated using membrane-immobilized enzymes.

\section{Acknowledgments}

This work was supported by JSPS KAKENHI Grant Number 25410145. The mass analysis of ACTH was carried out on a MALDI-TOF MS (Voyager-DE PRO) at the Division of Applied Protein Research, Advance Research Support Center, Ehime University.

\section{References}

[1] Samygina, V. R.; Moiseev, V. M.; Rodina, E. V.; Vorobyeva, N. N.; Popv, A. N.; Kurilova, S. A.; Nazarova, T. I.; Avaeva, S. M.; Bartunik, H. D. J. Mol. Biol. 2007, 366, 1305-1317.

[2] Gwyer, J. D.; Richardson, D. J.; Butt, J. N. Biochemistry 2004, 43, 15086-15094.

[3] Otlewski, J.; Jelen, F.; Zakrzewska, M.; Olesy, A. EMBO J. 2005, 24, 1303-1310.

[4] Vanchugova, L. V.; Valueva T. A.; Romashikin, V. I.; Rozenfel'd, M. A.; Valuev, L. I. Biokhimila 1988, 53, 1455-1461.

[5] Plancken, I. V.; Remoortere, M. V.; Loey, A. V.; Hendrickx, M. E. Biotechnol. Prog. 2004, 20, 82-86.

[6] Bolognesi, M.; Djinovic-Carugo, K.; Ascenzi, P. Monaldi Arch. Chest. Dis. 1994, 49, 144-149.

[7] Zhou, J. M.; Liu, C.; Tsou, C. L. Biochemistry 1989, 28, 1070-1076.

[8] Shimazaki, Y.; Kohno, Y. Anal. Biochem. 2012, 422, 55-57.

[9] Shimazaki, Y.; Kohno, Y.; Fukui, I.; Koyashiki, T. Protein Expr. Purif. 2012, 83, 177-181.

[10] Shul'gin, M. N.; Valueva, T. A.; Kestere, A.; Mosolov, 
V. V. Biokhimila 1981, 46, 473-480.

[11] Shimazaki, Y.; Hashimoto, A. Talanta 2014, 125, 400-404.

[12] Shimazaki, Y.; Sato, Y. J. Chromatogr. B 2016, 1021, 108-113.

[13] Lu, W.; Apostol, I.; Qasim, M. A.; Wame, N.; Wynn, R.; Zhang, W. I.; Anderson, S.; Chiang, Y. W.; Ogin, E.; Rothberg, I.; Ryan, K.; Laskowski, M. J. Mol. Biol. 1997, 266, 441-461.

[14] Vistoli, G.; Pedretti, A.; Mazzolari, A.; Testa, B. Bioorg. Med. Chem. 2010, 18, 320-329.

[15] Sakikawa, T.; Shimazaki, Y. J. Pham. Biomed. Anal. 2012, 71, 179-182.

[16] Bonetoo, V.; Bergmann, A. C.; Jornvall, H.; Sillard, R. Anal. Chem. 1997, 69, 1315-1319.

[17] Corthals, G. L.; Gygi, S. P.; Aebersold, R.; Patterson, S. D. In Identification of proteins by mass spectrometry in Proteome research, Two-dimensional gel-electrophoresis and identification methods; Rabilloud, T. Ed; Springer: Berlin, Heidelberg, 2000; Chapter 10.

[18] Ko, K. Y.; Ahn, D. U. Poult. Sci. 2008, 87, 1441-1450.

[19] Abeyrathne, E. D.; Lee, H. Y.; Ahn, D. U. Poult. Sci. 2014, 93, 1010-1017.

[20] Gjessing, E. C.; Emery, R.; Clements, J. P. J. Biol. Chem. 1959, 234, 1098-1101.

[21] Rhodes, M. B.; Azari, P. R.; Feeney, R. E. J. Biol. Chem. 1958, 230, 399-408.

[22] Waheed, A.; Salahuddin, A. Biochem. J. 1975, 147, 139-144. 